\section{GP112 LOOKING BEFORE WE YEEP: AN EVALUATION OF THE EXPERIENCES OF HEALTHCARE TRANSITIONS FOR YOUNG PEOPLE WITH 22Q11DS}

'Diarmuid Lynch* 1,2,3 Lorna Kerin, 1,4,5Fiona McNicholas. 'UCD, dublin, Ireland; ${ }^{2} T U S L A$, Dublin, Ireland; ${ }^{3}$ Loveknowledge Consultancy, Dublin, Ireland; ${ }^{4} \mathrm{OLCHC}$, Dublin, Ireland; ${ }^{5}$ Lucena Clinic SJOG, Dublin, Ireland

\subsection{6/archdischild-2019-epa.177}

Background The importance of effective and well managed transition care has been increasingly recognised along with the reality that for many it is poorly experienced. National and International policies have emphasized the importance of addressing these shortcomings. Individuals with the rare genetic disorder, 22q11.2 deletion syndrome (22Q11DS) face particular challenges with transition. By virtue of their genetic condition, they are at a highly increased risk of psychiatric disorders along with a variety of medical and educational problems, with the majority experiencing mild intellectual disability, all contributing to unique challenges of transition into adulthood. To date, the experience of these young people navigating this journey has not been well researched.

Aim This current project seeks to understand the experiences of a group of young people (Young Experts by Experience Panel, YEEP) as they journey from adolescences to adulthood, interfacing with many different healthcare providers. It seeks the views of YEEP as to methods to optimise this experience, and presents a practical transitional toolkit, designed by the YEEP.

Method Young people were recruited via the national support organisation following ethical approval. A semi-structured interview schedule was devised and guided participatory action research regarding transition experience and suggestions for future improvements.

Results The overwhelming experience by YEEP members was of a difficult and stressful transition, with poor transfer of information between services and an overall lack of knowledge by the new service providers of their unique needs and disorder (22Q11DS), requiring multiple retelling of their story. Their key recommendations was to develop a 'Transition Toolkit' to assist in the process, acting as a conduit of information from one professional to another, an aide memoire for themselves documenting key appointment dates and treatments and to which they could add useful information and resources. Details of the content/format of this will be presented.

Conclusion Optimum transition experience is critical to engagement with the new services, management of the medical condition and overall quality of life. Such care should be planned and co-ordinated, with the young person central to the process, and assisted by the family and clinical teams. Transition training for professionals coupled with the transition Toolkit, should assist in this endeavour.

Acknowledgements Support given by Ms Anne Lawlor, Chairperson of 22Q Ireland, and the members of the YEEP group which made the SSRA experience so enriching. This work was carried out as part of medical student elective. (DL 3rd year GEM -presenter)

\section{GP113}

CAREGIVER PERCEPTIONS OF A COMPLEX CARE SERVICE: IMPLICATIONS FOR SERVICE DEVELOPMENT

${ }^{1,2,3}$ Anita D'Aprano, ${ }^{1,4}$ Susie Gibb* , ${ }^{4}$ Elaine Meehan, ${ }^{1,2,4}$ Monica Cooper, ${ }^{1}$ Nicki Mountford, ${ }^{1}$ Suzi Riess. 'Royal Children's Hospital, Melbourne, Australia; ${ }^{2}$ University of Melbourne, Melbourne, Australia; ${ }^{3}$ Murdoch Children's Research Institute, Melbourne, Azerbaijan; ${ }^{4}$ Murdoch Children's Research Institute, Melbourne, Australia

\subsection{6/archdischild-2019-epa.178}

Background Changing medical care has resulted in growing numbers of Children with Medical Complexity (CMC). CMC have high care needs, often unmet by traditional healthcare models. In response to this need, the Complex Care Service (CCS) at The Royal Children's Hospital (RCH) in Melbourne was created. While there was preliminary pilot data on the level of parent satisfaction with the service, we did not have sufficient knowledge of the value and importance of the various components of the service or parent satisfaction as the service expanded.

Aim The aim of this study was to determine 1) what caregivers value most about the CCS service at the $\mathrm{RCH}$ and 2) explore caregiver perceptions of care.

Methods All caregivers of children who were enrolled in the CCS at RCH in April 2017 ( $n=103)$ were invited to participate. A purposefully designed survey explored their perceptions of care and included questions regarding: the extent to which the CCS improved patient quality of care; the extent to which the CCS components added value to patient care; satisfaction with the CCS; and likelihood of recommending the service to other families. Participants were invited to answer open ended questions about what the CCS did well, and what could be improved, and were invited to provide general comments.

Results Responses were received from 53 families (51\%). We found that 24 hour phone advice; coordination of appointments; a key contact; and access to timely information, are the most important components of a service for CMC for caregivers.

Overall, levels of satisfaction with the $\mathrm{RCH}$ service were high - over $90 \%$ of caregivers indicated that they were very/somewhat satisfied with care. Further, over $90 \%$ of participants perceived that the CCS improved their child's quality of care. Coordination, communication, family centred care, quality care and access were emergent themes in comments.

Conclusion This study provides important information for the design and operation of services for CMC in hospitals throughout Australia and further afield. Our findings highlight the importance of the key contact and family centred care This has implications for practice, as maintaining the quality of the service as it expands and is implemented more widely is a major sustainability challenge. It is crucial that we have a detailed understanding of what elements are required to support effective care coordination, to achieve successful implementation on a large scale. 